\title{
Advocating blended learning for university undergraduate level mathematical instruction beyond Covid-19
}

\author{
Haziq Jamil \\ Mathematical Sciences, Faculty of Science \\ Universiti Brunei Darussalam
}

\author{
Huda M Ramli \\ Mathematical Sciences, Faculty of Science \\ Universiti Brunei Darussalam
}

\author{
Elvynna Leong \\ Mathematical Sciences, Faculty of Science \\ Universiti Brunei Darussalam
}

August 20, 2021

\begin{abstract}
Institutional mathematics education has long been traditional in its ways of being teacher-centric, a tradition which perhaps dates back to the Ancient Greece. Much like the society in those days, where there was a wary public feeling about the rigidness of the mathematical instruction in Pythagoras' school, mathematics educators find themselves in a similar position in the common era of 2020. Unlike the Ancient Greece however, the battle is for the continuous delivery of a comprehensive mathematics education in the midst of the Covid-19 pandemic. It would be fair to say that mathematics departments across all levels of the education sector have been affected drastically; more so on instructors who prefer the traditional "chalk and talk" method of instruction. In this article, we share several lessons learned in the delivery of mathematical instruction at undergraduate university level during the Covid-19 pandemic, drawing on our experience at Universiti Brunei Darussalam. These include specific methods for implementing online learning effectively, the pros and cons of such methods, and how we can use computer based tools to make learning more conducive. We highly think that these implementations are beneficial to be adapted by mathematics departments anywhere as a means of adapting to the new realities post Covid-19.
\end{abstract}

Keywords: Blended learning, dynamic exercises, learning management systems, automated assessments, Covid-19

\section{Introduction}

Institutional mathematics education has long been traditional in its ways of being teachercentric, a tradition which perhaps dates back to the Ancient Greece. Writing out a mathematical narrative on the board while talking aloud is reported to be the central 
pedadogical genre of the undergraduate mathematics lecture classroom (Artemeva \& Fox, 2011). One almost yearns that the romantic picture of Pythagoras gathering his students in the piazza to demonstrate one of his theorems, as portrayed in Raphael's The School of Athens, can be proven to be true. In any case, the methods of old are still seen to this day especially for university level teaching (Fauzan et al., 2002). Mathematicians continue in this style of teaching, partly to honour the long standing traditions of Ancient Greek pedagogy, and in another part believing it to suffice. All that is needed for mathematics is an open mind.

The coronavirus disease 2019 (Covid-19) pandemic in Brunei Darussalam is an evolving situation with extraordinary challenges for staff and students of Universiti Brunei Darussalam, UBD (Universiti Brunei Darussalam, 2020). On 9 March 2020, the first case of Covid-19 infection was reported in Brunei Darussalam (Ministry of Health, 2020). All lectures and tutorials at UBD were transitioned to online platforms from 12 March 2020 to minimize face-to-face interactions on campus. Face-to-face lessons, which include small group tutorials and discussions, resumed on 18 June 2020 when situation gradually returned to normal. However, lectures were still conducted online. The University then implemented blended learning for all the modules, which is a blend of offline and online instruction, from 27 July 2020 where students experience both physical and online class. Lectures continued to be delivered online (Universiti Brunei Darussalam, 2020). UBD requires their lecturers to have atleast a minimum of $40 \%$ online activities as part of their teaching and learning method. These measures were enacted by UBD as a result of the ongoing strategies in controlling the Covid-19 outbreak in the country.

The implementation of blended learning in higher education is increasing (Boelens et al., 2018), often with the aim to offer flexibility in terms of time and place to a diverse student population (Graham et al., 2013). A study revealed that using blended learning approach improved students' mathematical achievement scores as compared to fully online and face-to-face learning (Awodeyi et al., 2014). The many advantages of incorporating an online component to learning is not unknown, with many other authors being proponents of such methods, producing research articles on the subject even before the pandemic era (Ahmad et al., 2008; Borba et al., 2016; Lin et al., 2016; Yushau, 2006; Quinn \& Aarão, 2020; Setyaningrum, 2018). For mathematics in particular, positive effects due to blended learning have been recorded (Ahmad et al., 2008). In that paper, they looked at how supplementing face-to-face teaching with a computer-based courseware improved students understanding on the topic of integration. Blended learning also helps students in understanding mathematical concepts better (Setyaningrum, 2018). This is in part due to the fact that students have far better control of the pace and regularity in which they access the learning material. Another quasi-experimental research showed that certain subgroups of students fare better with blended learning 
approaches for matheamtics subjects (Lin et al., 2016). Not only that, in that study the authors noted a positive perception towards blended learning in general, but also an increased uptick in the use of learning management systems (LMS) such as Moodle or Canvas. This suggests that the success of the adoption of LMS is tied to the curriculum design itself. On another note, if anything, the blended learning approach has a secondary objective of making students be more familiar with computing technology (Yushau, 2006), something that is quite pertinent in this digital day and age of IR 4.0.

Locally at UBD, the blended learning approach seems to have support among the student body as well. Each academic semester, UBD invites students to complete a student feedback exercise regarding the aspects of the course delivery such as material, pace, seminar activities, and so on. The open-ended comments specifically from mathematics students are discussed here, coming from students who had essentially experienced both the "regular" style of traditional learning, and also the online approach immediately after the Covid-19 restrictions were made in place. Interestingly, the theme of negative comments from students did not revolve around difficulties of online learning. That is to say, the same kinds of problems were noted such as "exercises are too difficult", or "please give more examples"-all of which would've been the same feedback had the classes been fully offline. In contrast and rather unexpectedly, the acceptance of an online based approach to teaching mathematics is certainly higher than anticipated. Students found that, for example, with pre-recorded lectures and having access to the videos, they were much more able to follow along the lectures than having it face-to-face. The main reason for this is that they have the ability to rewind and rewatch the lectures at their own pace. When English is not their main language, this provides an additional advantage as they are then able to consult dictionaries and the like to be able better comprehend their instructors. This seems to be backed by the independent survey results conducted by Shahrill et al. (2020), who suggested that $93.3 \%$ of students responded that university and faculty activities during the outbreak were acceptable, good, very good or excellent. There is clearly a backing from the student body to maintain an online element in the curriculum.

While all of this seems to be campaigning for the total elimination of the traditional chalk and talk method of instruction, we should not forget that there are valuable skills to be learnt as well. For one, the writing of mathematics itself is a skill perfected through repetition and exposure. By 'writing' we mean both the aspects of penmanship (indeed, it could be argued that the first step in mathematics communication is the correct symbolisation of the concepts) and argumentation \& logic. For another, students learn to summarise the appropriate knowledge 'on-the-fly', thereby practicising the ability to multi-task (listening as well as writing notes). The act of writing itself aids in memory retention (Abu-Rabia, 2003), while the availability of a single source of reference (students' 
notes) is a passive method of requiring students to venture beyond this (e.g. notes review, book chapter readings, peer discussions, etc.). Although, while this method of teaching suits the more independent of students, it is good training for students to become more independent because of it. The blended-learning approach really aims to capture the best of both online and offline approaches to teaching.

Our paper highlights the many advantages of online-based instruction for mathematics, and consequently advocates blended learning approaches as a way forward even beyond the pandemic era. We shall touch on the various approaches to online instructing (specifically for mathematics subjects at undergraduate university level), and share what works and what doesn't from our experience during the Covid-19 pandemic. Section 2 highlights various instructional strategies, particularly on conducting lectures and tutorials online. Section 3 discusses conducting online based assessments and how the use of an $\mathrm{R}$ package can help streamline the process. Finally, we conclude in Section 4 with recommendations on the appropriate composition of online and offline teaching.

\section{Instructional strategies}

Blended learning is a pedagogical combination of direct contact between lecturers and students and online instruction using various digital platforms. There are several options for online instruction such as lectures, discussions, demonstrations, simulations, games, problem-based learning projects, case studies-to name a few. In the course design of a mathematics module, we find that the first four modes make the best combination of an instructional strategy.

Online lectures are the best way to introduce the main mathematical concepts of the course to students. Lectures can be delivered synchronously (live lectures where students and lecturer conference at the same time) or asynchronously (using prerecorded lecture videos). In synchronous mode, discussions and demonstrations can follow during the live lectures. Whereas in asynchronous mode, demonstrations of the mathematical processes should be included in the recorded lecture and offline discussions can be posted in LMS (e.g. Canvas and Moodle) forums. UBD subscribes to Canvas, the official learning management system (LMS) for the University, and has been in practice since 2014 for teaching and learning activities, particularly in online and blended learning modes (Shahrill et al., 2020).

Using this student-centred pedagogy, the lecturer is able to give instructions to students via readings or lecture slides that have been shared beforehand. The contents of the lecture slides should be minimal so that the lecturer can elaborate on the concepts further during the live or recorded lecture. To illustrate this point, let's consider 
a simple topic on Gaussian elimination. The fundamental idea is to find solutions of a linear system of equations by adding multiples of one equation to the others, in order to eliminate a variable. We continue this process until only one variable is left. This is a very simple concept that students can absorb during the lecture. However, applying this process in the linear algebra context may become challenging to some students. In the online lecture, the lecturer could start by writing down on the slide presentation or smartboard, the expression of a given linear system in matrix form: $A \mathbf{x}=\mathbf{b}$, followed by an explanation of the different terminologies namely coefficient matrix, augmented matrix and elementary row operations. Finally, it is key that the lecturer spend some time to further demonstrate the process of Gaussian elimination through a step-by-step guided example of solving a linear system.

An online instruction such as described above that includes demonstration instead of just talking through the slide presentation, students are able to learn and capture the mathematical thinking behind the process just like they would be able to in a traditional classroom. Offline, students can work independently on the contents that require simple reasoning and memory. Self-motivated learners who analyze, speculate and explore the problems independently will be able to achieve a deeper understanding of the concepts. This method of teaching allows the instructor to guide the students to progress steadily and instill the attitude of independent study and creativity.

During the peak of the outbreak when direct contact with students was totally restricted at UBD, online lectures received significantly positive responses from the students as opposed to online tutorial classes. Unlike an instructional lecture session where the instructor is expected to present the learning materials, tutorial classes more often than not require an active engagement from the students. For an average mathematics student with introverted tendencies, it can be quite difficult for a lecturer to facilitate this engagement.

Tutorials do not work online. The idealistic way of conducting this online, is if everyone had a tablet and they can share their screen. This replicates them writing their answers on the whiteboard in class. But not all students have tablets (in fact a vast majority do not), so this was very unfeasible to do. In the end, instructors called upon students for their solutions and the best they could do is describe their solutions orally or angle their video cameras to their paper writing pads (assuming their internet connection was stable enough to deliver a high fidelity image).

Fortunately, after 18 June 2020 restrictions were gradually lifted in the nation and face-to-face tutorials are now allowed at UBD. Since then, we found that face-to-face tutorials to be a tremendous support alongside online instructions for the students. The offline tutorials or problem classes help to construct knowledge and develop a better 
understanding of the mathematical processes, especially for individual students who encounter difficulties in self-paced learning. To encourage more collaboration and independent study, the flipped classroom approach is a great way to allow active group dynamics. Flipped classroom (sometimes referred to as reverse classroom) is a type of blended learning where students are given standalone module readings or online resources to work on independently before in-class activities. Face-to-face class activities may include a guided or unguided skill practice, group discussions, presentations, games or quizzes and peer assessments.

For a successful blended learning environment, instructors need to consider the best instructional strategy to combine online and face-to-face activities (Schneider \& Preckel, 2017). It can be challenging to find an adequate substitute for the live lecture, so it helps to have a few guidelines in mind while designing a blended learning course.

Understand learning styles: We find that each cohort tend to have different learning styles; self motivated versus group motivated. Understanding their learning styles earlier on is beneficial in designing the course's contact activities accordingly, in order to optimise the cohort's learning outcomes. Mathematics anxiety amongst mature mathematics learners is a real issue that results in avoidance behaviours, such as putting off doing mathematics work until the last minute. This characteristic could be eased when students are allowed to self-learn in the privacy of an online environment (Marshall et al., 2017).

Keep it as simple as possible: Learning mathematics can be quite intimidating especially for first-year students who haven't developed the proper study techniques to be successful at university-level mathematics. For this reason, online learning materials should be kept as simple as possible to support the process of learning, as students develop a positive perception of the mathematical context. It wouldn't hurt to assume that the students know nothing to begin with. Repeating information (particular labels, acronyms or terminology) is good and necessary to prevent ambiguity.

Get organized: Prior to the course start, it is crucial that the students understand the outline and learning outcomes for each chapter of the module. It is a good idea to spend a few minutes of the first lecture explaining the objectives of the module, especially student's expectations to complete the module. These information should also be readily available within the LMS so that students can refer back, should they need to. Follow the teaching plan and module learning outcomes, as expected from the students.

Be compassionate: Last but not least, try to understand the students and discuss their comfort level in using technology. Do they have access to computers in their household? Do they have good internet connection? We are stepping into a "new norm" in digital 
teaching and individual learners may need extra support in the online environment. Be patient and always have a backup plan. For example, lecturers can set up a different communication channel (e.g. Telegram) in case of any technological hiccup.

\section{Assessments}

Here we discuss the pros and cons of implementing online-based assessment for mathematics, particular online quizzes and open-book examinations. The concept of online tests via MCQs or the like is not new, and instructors who already make use of learning management systems such as Canvas or Moodle should be familiar with the features within the LMS to implement these. Nonetheless, the irritation here is two-fold. Firstly, the actual writing of the assessment questions takes time because typesetting equations in the native learning management system (LMS) feature set is very clunky. This would be a hindrance and possible inadequate workaround to this would be to screenshot equations from other ${ }^{A} T_{\mathrm{E}} \mathrm{X}$ typesetting sources (e.g. online $\mathrm{LT}_{\mathrm{E}} \mathrm{X}$ ) or worse still abandon $\mathrm{H}_{\mathrm{E}} \mathrm{X}$ equations and type the "equation" as is e.g. typing $\mathrm{E}\left(\mathrm{X}^{\wedge} 2\right)$ to denote $E\left(X^{2}\right)$. This may lead to confusion or reluctance to ask questions relating to complex equations. Secondly, there is always a worry that students may cheat during online assessments. Common issues that arise here are that students have access to teaching material or Google, they may collaborate among themselves, and issues with time management (from the instructors perspective, this is about unsure what a good amount of time is needed for the assessment).

We have found a very simple and straightforward way of overcoming the first problem, and that is by way of an $R$ package called exams (Grün \& Zeileis, 2009). The package allows for writing assessment questions either in Markdown or $\mathrm{LT}_{\mathrm{E}} \mathrm{X}$ (Sweave) format documents, which are then converted to a variety of output files. For example, a singular question file (.md or .Rnw) can be exported as a PDF document, an HTML file for viewing on the web, or most importantly, to other LMS such as Canvas or Moodle. It is possible to produce multiple choice type questions (single or multiple correct answers) as well as questions which require a numeric answer. Technically it is also possible to write open-ended questions, however these may not be automatically graded.

On the subject of writing questions, we highlight one key feature of the exams package which enriches the assessment experience, and that is this concept of dynamic tests. It is possible to write questions such that each student gets a set of questions that is unique to him or her. For numeric answer questions, this involves $\mathrm{R}$ generating a different set of values for the student to process, and thereby requiring a different correct answer for each student. One example of this is on the topic of hypothesis test of equivalence of means in two independent samples. The question may read as follows: 
Let $X_{1}, \ldots, X_{n}$ be a random sample from $N\left(\mu_{x}, \sigma_{x}^{2}\right)$, and let $Y_{1}, \ldots, Y_{n}$ be a random sample from $N\left(\mu_{y}, \sigma_{y}^{2}\right)$. Calculate the value of the observed test statistic for testing $H_{0}: \mu_{x}=\mu_{y}$ against the alternative $H_{1}: \mu_{x} \neq \mu_{y}$. Do you reject the null hypothesis at the $5 \%$ level?

Students are also provided a sample data set ( $n$ is not too large here) from the following $\mathrm{R}$ code:

$\mathrm{x}<-\operatorname{rnorm}(8$, mean $=1.0, \mathrm{sd}=1)$

$\mathrm{y}<-\operatorname{rnorm}(8$, mean $=2.0, \mathrm{sd}=1)$

What is interesting is that each student will be presented with a completely different data set, as the exams package will use a different random seed for each student. On a technical note, the power of the independent two-sample $t$-test is known to depend on the sample size, so with $n=8$ and such an effect size the power is low enough that the incorrect null hypothesis is not be rejected some times, and other times it will be.

For multiple-choice type questions, one may provide an answer list that is longer than the number of possible choices. For instance, for a 4-choice MCQ, one may provide an answer list of eight choices. The exams package will then randomly select four out of the eight possible choices differently for each student. Of course, depending on the number of students, some students may in fact get the same choices for the question. This can be further enhanced by having the answer list permuted randomly for each question. This goes a little way into ensuring the integrity of the test by minimising the "ease of cheating" in the test.

As mentioned, the exams package allows the assessment to be exported to a variety of file types. This is pertinent to how the instructor wishes to conduct the assessment. If the test is to be conducted in a live classroom, then the test can be converted to a PDF document. The template of the PDF document can even be customised to include things such as the university name, module code, instructions to students, and so on. Moreover, the exams package will create a unique "paper id" for each paper generated. The instructor then must keep track of which student received which paper id, so that this may be traced back to the correct answer key that the package generates. This is crucial so as to facilitate the marking of answer scripts (since each script's correct answers will be different!), but this step can easily be done, for e.g., the instructor could arrange a seating plan for the students so that each seat corresponds to a known paper id. Incidentally, for MCQ type questions, it is possible to have the exams package generate an answer sheet which is then able to be read by optical charachter recognition (OCR) technology within the package itself. This would definitely go a long way to save time in marking the students' assessments. This also means that generating exam questions is 
sustainable even for large number of students (100-200 class size).

The best feature in our opinion is the ability for the exams package to export the same files and questions to a format that is recognisable by Canvas or Moodle. Other learning management platforms are possible such as Blackboard, OLAT, and Ilias. Of course, such assessments will be automatically graded, unless open-ended questions are posed to students.

Now we discuss the validity of such assessments (i.e. multiple choice questions as well as numeric answer questions, where the emphasis is more on the correct answer rather than the working and train of thought to arrive at the answer) for mathematics. Opponents of such methods of assessment argue that the lack of focus on the art of problem solving does nothing to hone students' logical thinking, which is in complete contravention of the ideal learning outcomes for a mathematics degree. Further, these kinds of questions have a zero-tolerance to mistakes, which brings about disillusion towards one's mathematical abilities, especially for those students who actually had the right approach to a question but made a single fatal error in their calculations. Tao (2008) in his insightful blog post also wrote that if a student arrives at answer which is not part of the list of available choices, they will then proceed to "fudge" their calculations in order to arrive at one of the most least objectionable choices on the list. This is a bad thing indeed to instill in budding mathematicians.

Despite all of their shortcomings, proponents of MCQ type questions argue that the role of such assessments is not one that is the primary method of assessing students' knowledge, but rather in a supporting capacity. When used correctly, it can provide valuable instant feedback and intervention for students who originally have a flawed understanding of a particular mathematics concept. Furthermore, in the spirit of Ebinghaus' forgetting curve (Murre \& Dros, 2015), the more exposure students get to the same concept, the better the memory retention. This would require a lot of effort in a traditional setting (e.g. conducting offline topical written tests, which would need to be marked), but in a blended-learning approach, this is practically seamless.

As a case example in one of our advanced statistics courses, we use MCQ online (Canvas) quizzes to implement online topical tests (seven quizzes in total spanning a period of 14 weeks, and the weight of these quizzes is $20 \%$ of the entire module). The two primary intention of these quizzes is to reinforce the students' learning and for instructor to gauge their understanding of the topics taught. Each quiz comprises of about $15 \mathrm{MCQ}$ questions, where students choose from a list of five possible choices. Students have 12 hours to complete the quiz, so as to take the pressure of time constraint away from the evaluations. This gives students ample time to refer back to the lecture slides, videos and notes, and in doing so achieves the objective of reinforcing their learning. At the same 
time, care is taken so that the questions actually address gauging their understanding. This means that there is less of an emphasis on fielding questions which requires one to calculate, but rather questions are more on testing definitions and logic. Here is an example question.

Q1: Which of the following statements best describes a point estimator?

1. It is the average of the sample variance

2. It is a single value that is the best estimate of an unknown sample statistic

3. It is a single value that is the best estimate of an unknown population parameter

4. It is the average of the population values

5. It is a number which can be used to estimate a point in time which is unknown

This particular question actually tests the students' understanding of the concept "point estimator". This one was interesting because there was no direct definition provided for point estimator (and hardly any statistics text book gives this definition). Instead, students surmise this fact from paragraphs describing the activity of point estimation and what the outcome of such activities are. In the lecture videos and handout slides, there was a single point of reference for the students regarding this, and that was a slide describing the concept of random variables being drawn from a parametric family with parameter $\theta$, and based on the samples obtained how one might deduce the most plausible value for the unknown $\theta$.

It should be noted that more straightforward type of definition questions can be asked too, with the intention of highlighting that particular area of the topic. For instance, in point estimation, there is a large focus on the properties of estimators that are derived, so one might ask

Q2: The correct definition for the variance of an estimator $\hat{\theta}$ is...

1. $E\left[(\hat{\theta}-E(\hat{\theta}))^{2}\right]$

2. $E\left[(\hat{\theta}-\theta)^{2}\right]$

3. $E[\hat{\theta}-E(\hat{\theta})]$

4. $\sqrt{E\left[(\hat{\theta}-E(\hat{\theta}))^{2}\right]}$

5. $\sqrt{E\left[(\hat{\theta}-\theta)^{2}\right]}$ 
With the choice of answers including similar form to the correct answer (choice 1) but with a little bit of tweaking. These would be confusing enough for them to double-check their answers in the relevant parts of the course material, thereby achieving the objective of reinforcing learning.

Another kind of question is one that requires careful examination of the entire list of choices to decide which one is correct. This lends itself to an extension of a 'TRUE/FALSE' type question to multiple choices. For instance, on the topic of convergence of random variables, one could ask to pick out the correct statement from a list of possible choices regarding the central limit theorem:

Q3: The following statements about the central limit theorem are all true except...

1. The standardised sample mean converges in distribution to the standard normal distribution

2. The limiting distribution of the random variables is normal regardless of the parent distribution

3. The CLT implies that the sample mean "piles up" around the true mean as more and more samples are collected

4. The CLT allows us to make approximate probabilistic statements about the sample mean for finite $n$

5. The CLT allows us to gather about the variability of the sample mean

This way again, with the intention of reinforcing their learning, students will refer to the relevant sections in their notes or lectures before deciding on the correct choice. The general feedback from students is that these kinds of questions are the trickiest to handle, simply because there are usually only subtle differences between the choices given and thereby making it quite difficult to pick out the supposedly correct answer. Arguably, to field these kinds of questions during a time-limited exam would be quite unfair (due to time pressure) and practically useless-we would only be differentiating between students who truly knows the material well and those who are completely clueless, but not a student who lies in between. It is only through these online type quizzes that such questions can be administered, providing students an invaluable opportunity to correct any misconceptions that they have regarding the study material. 


\section{Conclusions}

The Covid-19 pandemic really forced lecturers and students alike to step out of their comfort zones, and to take forward-looking and innovative approaches to maintain the delivery of mathematical instruction. One thing that is undeniable is that the pandemic hit the world at a time when technological landscape is favourable, requiring everyone to scramble and explore ways in which technology can help with learning. While the initial part of the journey may have been rough as hardly anyone was prepared for such a drastic change of plans to the education process, through a process of trial-and-error and thoughtful reflection, we share our recommendations for a blended learning approach to teaching mathematics at university level.

Firstly, with proper planning mathematics lectures and demonstrations can be successfully conducted online. It is essential that the lecturer take the time to understand the students' learning styles and deliver the online instructions accordingly, in order to encourage self-regulation of learning. Plan for students' engagement by preparing discussion topics and quizzes, synchronously or asynchronously. Tutorials on the other hand, should remain offline whenever possible. Unless all the students possess all the necessary equipment (such as tablets and digital pens) to replicate writing on a whiteboard as done in a traditional classroom, it is not feasible to facilitate an interactive learning experience for online tutorials. Face-to-face tutorials will serve as a substantial support of mathematical thinking in the blended learning environment.

Secondly, the blended approaches to lecturing and tutorials can be complemented with a blended approach to assessments. As a mathematics instructor it is worthwhile to take a step back and reflect on what the learning objectives are for a particular course, and then implement the online and offline assessments accordingly. In general, we feel that the two themes of mathematics are assessment are on understanding (do students understand the concepts presented?) and replication (will students be able to answer a previously unseen but similar question?). On the theme of understanding, we argue that online quizzes (either MCQ style or numeric solutions) provide an indispensable element of solidifying and reinforcing students learning of various mathematical notions. Online quizzes are self-grading (when used with an LMS) and easily scalable to the largest of classes. When designed correctly, these online assessments give lecturers feedback too regarding the level of understanding of the class and allows for appropriate intervention to be made.

Of course, online quizzes only provide one aspect of assessment, so must be supplemented with other forms of assessments, in particular to gauge the level of knowledge reproducibility. For this, traditional forms of assessment such as exercise sheets are still recommended to be included in the curriculum. The exercise sheet loads may be reduced 
in quantity or in weight, especially if the online quizzes are weighted as well. Reduction in exercise loads reduce the burden on instructors to mark such assessments too. We do not feel that this adversely affects learning if implemented with an online component. One strategy that works well in our experience is having summative assessment weights equal to $40 \%$, with equal weights being contributed by online quizzes and exercise sheets.

Throughout this paper we avoided a discussion about exam implementation. The main reason for this is that often times the decision on the method of examination may fall out of the instructors and lecturers hands. At UBD for instance, as a policy $60 \%$ weight is given to examinations as standard, and during the pandemic the choice given to instructors was whether to conduct the exam online (open-book) or to convert the exam component to a full assessment module (e.g. coursework). Nonetheless, this paper is meant to advocate for blended learning approaches beyond the Covid-19 pandemic, so with this in mind, the traditional time-based examinations may well return so may be administered as usual. This would certainly go well with the blended approaches suggested above.

\section{References}

Artemeva, N., \& Fox, J. (2011). The writing's on the board: The global and the local in teaching undergraduate mathematics through chalk talk. Written Communication, $28(4), 345-379$.

Fauzan, A., Slettenhaar, D., \& Plomp, T. (2002). Traditional mathematics education vs. realistic mathematics education: Hoping for changes. Proceedings of the 3rd International Mathematics Education and Society Conference, 1-4.

Universiti Brunei Darussalam. (2020). Ubd in the covid-19 pandemic. https://ubd.edu. bn/news-and-events/covid19.html (accessed 5 March 2021)

Ministry of Health. (2020). Detection of the first case of covid-19infection in brunei darussalam. http: / / www . moh.gov. bn / Shared \% 20Documents / 2019\% 20ncov / press\%20releases /FINAL\%20Press\%20Release\%20(eng)\%20-\%20First\%20Case\% 20COVID - $19 \%$ 20in \% 20Brunei \% 20Darussalam \% 20(2).pdf (accessed 8 March 2021)

Boelens, R., Voet, M., \& Wever, B. D. (2018). The design of blended learning in response to student diversity in higher education: Instructors' views and use of differentiated instruction in blended learning. Computers and Education, 120, 197-212.

Graham, C. R., Woodfield, W., \& Harrison, J. B. (2013). A framework for institutional adoption and implementation of blended learning in higher education. Internet and Higher Education, 18, 4-14. 
Awodeyi, A. F., Akpan, E. T., \& Udo, I. J. (2014). Enhancing teaching and learning of mathematics: Adoption of blended learning pedagogy in university of uyo. International Journal of Science and Research, 3(11), 40-45.

Ahmad, W., Shafie, A. B., \& Janier, J. B. (2008). Students' perceptions towards blended learning in teaching and learning mathematics: Application of integration. Proceedings 13th Asian Technology Conference in Mathematic (ATCM08), Suan Sunanda Rajabhat, University Bangkok, Thailand.

Borba, M. C., Askar, P., Engelbrecht, J., Gadanidis, G., Llinares, S., \& Aguilar, M. S. (2016). Blended learning, e-learning and mobile learning in mathematics education. $Z D M, 48(5), 589-610$.

Lin, Y.-W., Tseng, C.-L., \& Chiang, P.-J. (2016). The effect of blended learning in mathematics course. EURASIA Journal of Mathematics, Science and Technology Education, 13(3), 741-770.

Yushau, B. (2006). The effects of blended e-learning on mathematics and computer attitudes in pre-calculus algebra. The mathematics enthusiast, 3(2), 176-183.

Quinn, D., \& Aarão, J. (2020). Blended learning in first year engineering mathematics. ZDM, 52(5), 927-941.

Setyaningrum, W. (2018). Blended learning: Does it help students in understanding mathematical concepts? Jurnal Riset Pendidikan Matematika, 5(2), 244-253.

Shahrill, M., Petra, M. I., Naing, L., Yacob, J., Santos, J. H., \& Aziz, A. B. Z. A. (2020). New norms and opportunities from the COVID-19 pandemic crisis in a higher education setting: Perspectives from Universiti Brunei Darussalam. International Journal of Educational Management.

Abu-Rabia, S. (2003). The influence of working memory on reading and creative writing processes in a second language. Educational psychology, 23(2), 209-222.

Schneider, M., \& Preckel, F. (2017). Variables associated with achievement in higher education: A systematic review of meta-analyses. Psychological Bulletin, 143(6), 565-600.

Marshall, E. M., Staddon, R. V., Wilson, D. A., \& Mann, V. E. (2017). Addressing maths anxiety within the curriculum. MSOR Connections, 15(6), 28-35.

Grün, B., \& Zeileis, A. (2009). Automatic generation of exams in r. Journal of Statistical Software, Articles, 29(10), 1-14. https://doi.org/10.18637/jss.v029.i10

Tao, T. (2008). On multiple choice questions in mathematics. https://terrytao.wordpress. com / 2008/12/14/ on- multiple- choice- questions-in-mathematics/ (accessed 6 March 2021)

Murre, J. M., \& Dros, J. (2015). Replication and analysis of ebbinghaus' forgetting curve. PloS one, 10(7), e0120644. 